Proceedings of the Edinburgh Mathematical Society (2004) 47, 289-296 (C)

DOI:10.1017/S0013091503000166 Printed in the United Kingdom

\title{
REMARKS ON IMMERSIONS IN THE METASTABLE DIMENSION RANGE
}

\author{
CARLOS BIASI ${ }^{1}$ AND ALICE KIMIE MIWA LIBARDI ${ }^{2}$ \\ ${ }^{1}$ Departamento de Matemática, ICMC-USP-Campus de São Carlos, \\ Caixa Postal 668, 13560-970 São Carlos, SP, Brazil (biasi@icmc.usp.br) \\ ${ }^{2}$ Departamento de Matemática, IGCE-UNESP, 13506-700, \\ Rio Claro, SP, Brazil (alicekml@rc.unesp.br)
}

(Received 7 March 2003)

\begin{abstract}
In this work we present a generalization of an exact sequence of normal bordism groups given in a paper by H. A. Salomonsen (Math. Scand. 32 (1973), 87-111). This is applied to prove that if $h: M^{n} \rightarrow X^{n+k}, 5 \leqslant n<2 k$, is a continuous map between two manifolds and $g: M^{n} \rightarrow B O$ is the classifying map of the stable normal bundle of $h$ such that $(h, g)_{*}: H_{i}\left(M, \mathbb{Z}_{2}\right) \rightarrow H_{i}\left(X \times B O, \mathbb{Z}_{2}\right)$ is an isomorphism for $i<n-k$ and an epimorphism for $i=n-k$, then $h$ bordant to an immersion implies that $h$ is homotopic to an immersion. The second remark complements the result of C. Biasi, D. L. Gonçalves and A. K. M. Libardi (Topology Applic. 116 (2001), 293-303) and it concerns conditions for which there exist immersions in the metastable dimension range. Some applications and examples for the main results are also given.
\end{abstract}

Keywords: bordism; normal bordism; immersion of manifold; localization

2000 Mathematics subject classification: Primary 57R42

Secondary 55Q10; 55P60

\section{Introduction}

Let $h: M^{n} \rightarrow X^{n+k}$ be a continuous map from a closed smooth connected $n$-manifold into a smooth connected $(n+k)$-manifold, $5 \leqslant n<2 k$. Let us assume that $h$ is bordant to an immersion, in the sense of Conner and Floyd [4], and let $g: M \rightarrow B O$ be the classifying map of the stable normal bundle, $h^{*}\left(\tau_{X}\right) \oplus \nu_{M}$, of $h$, where $\tau_{X}$ denotes the tangent bundle of $X$ and $\nu_{M}=-\left(\tau_{M}\right)$. One may ask on which conditions of $(h, g)$ is $h$ homotopic to an immersion?

Let $f: M \rightarrow N$ be a continuous map between two closed smooth connected $n$-dimensional manifolds and suppose that $N$ immerses in $\mathbb{R}^{n+k}$, for some $k$, with $5 \leqslant n<2 k$. Under which conditions on $f$ does $M$ immerse in $\mathbb{R}^{n+k}$ ? The case when $M$ immerses in $\mathbb{R}^{n+k}$ and in which one is looking for conditions on $f$ such that $N$ also immerses in $\mathbb{R}^{n+k}$ has been considered in [2] and [5-7].

For both problems, we use a normal bordism approach $[\mathbf{9}]$, and give an answer in terms of the induced maps of $\mathbb{Z}_{2}$-homology groups. 
We prove the following main results.

Theorem A. Let $h: M^{n} \rightarrow X^{n+k}$ be a continuous map from a closed smooth connected $n$-manifold into a smooth connected $(n+k)$-manifold, $5 \leqslant n<2 k$, and let $g: M \rightarrow B O$ be the classifying map of the stable normal bundle of $h$. Given

$$
(h, g): M \rightarrow X \times B O,
$$

suppose that the induced map

$$
(h, g)_{*}: H_{i}\left(M, \mathbb{Z}_{2}\right) \rightarrow H_{i}\left(X \times B O, \mathbb{Z}_{2}\right)
$$

is an isomorphism for $i<n-k$ and an epimorphism for $i=n-k$.

Then if $h$ is bordant to an immersion, $h$ is homotopic to an immersion.

Theorem B. Let $M$ and $N$ be closed connected n-manifolds and let $f: M \rightarrow N$ be a continuous map such that

$$
f_{*}: H_{i}\left(M, \mathbb{Z}_{2}\right) \rightarrow H_{i}\left(N, \mathbb{Z}_{2}\right)
$$

is an isomorphism for $i \geqslant 0$.

Then if $N$ immerses in $\mathbb{R}^{n+k}$ for $5 \leqslant n<2 k$, so does $M$.

The paper is divided into four sections. In $\S 2$ we present two exact sequences of bordism groups. One of them is a generalization of the exact sequence of normal bordism groups given by Salomonsen [13]; it will be applied to prove Theorem A.

In $\S 3$ we prove Theorems A and B and in $\S 4$ we present an application of Theorem B by using a non-standard obstruction theory, and we give some examples for Theorem A.

In this work, $\mathcal{C}$ will denote the class of all torsion groups where the torsion is odd.

\section{Exact sequences of bordism groups}

In this section we generalize an exact sequence given in [13], by using identifications of some normal bordism groups.

Given a topological space $X$ and a virtual bundle $\phi$ over $X$ (i.e. an ordered pair of vector bundles $\phi^{+}$and $\phi^{-}$over $X$, written $\left.\phi^{+}-\phi^{-}\right)$, the $n$th normal bordism group of $X$ with coefficient $\phi$, denoted by $\Omega_{n}(X, \phi)$, is the bordism group of pairs $(h: M \rightarrow X, g)$, where $g$ is the stable bundle isomorphism $\tau_{M} \oplus g^{*}\left(\phi^{-}\right) \simeq \varepsilon^{n} \oplus g^{*}\left(\phi^{+}\right)$and $\varepsilon^{n}$ denotes the trivial bundle of dimension $n$. We recall that $\Omega_{n}(X, \phi)=\Omega_{n}\left(X, \phi+\varepsilon^{r}\right)$, and if $\phi$ can be expressed in the form $\phi=\varepsilon^{l}-\left(\phi^{-}\right)^{l}$, there is an isomorphism $\Omega_{n}(X, \phi) \simeq \pi_{n+l}^{S}\left(T\left(\phi^{-}\right)\right)$, where $T\left(\phi^{-}\right)$is the disjoint union of the (total space) $\phi^{-}$and a point $\infty$. For more details see $[\mathbf{1 3}]$ or $[\mathbf{9}]$. We adopt the Salomonsen convention.

Let us now consider $X$, an $(n+k)$-manifold, and let $\nu_{X}^{p}=-\left(\tau_{X}\right)$ be the stable normal bundle of $X$, with $p$ large enough. If $\phi^{p+k}=\varepsilon^{p+k}-\nu_{X}^{p} \times \gamma^{k}$, an element of $\Omega_{n}\left(X \times B O(k), \phi^{p+k}\right)$ can be considered as $\left[(h, g): M^{n} \rightarrow X \times B O(k), H\right]$, where

$$
H: \tau_{M} \oplus h^{*}\left(\nu_{X}^{p}\right) \oplus g^{*}\left(\gamma^{k}\right) \rightarrow \varepsilon^{p+k} \oplus \varepsilon^{n}
$$


is a stable bundle isomorphism and $g$ is the classifying map of the stable normal bundle of $h$. This is equivalent to the isomorphism $\nu_{M} \simeq h^{*}\left(\nu_{X}^{p}\right) \oplus g^{*}\left(\gamma^{k}\right)$ and, since $\nu_{X} \oplus \tau_{X}$ is trivial, $h^{*}\left(\tau_{X}\right) \oplus \nu_{M} \simeq g^{*}\left(\gamma^{k}\right) \oplus \varepsilon^{p+n}$. In this case, the stable normal bundle of $h$ has an $O(k)$-structure and then, by Hirsch $[\mathbf{8}], h$ is homotopic to an immersion. Let us denote $\Omega_{n}\left(X \times B O(k), \phi^{p+k}\right)$ by $I_{n}(X)$ and let $\mathcal{F}: I_{n}(X) \rightarrow \eta_{n}(X)$ be the forgetful map. We remark that if $[M, f] \in \eta_{n}(X)$ is an element of $\mathcal{F}\left(I_{n}(X)\right)$, then $f$ is homotopic to an immersion.

Let $\psi=\psi^{+}-\psi^{-}$be a virtual bundle over $X$. We note that the geometric dimension $g \operatorname{dim}(\psi) \leqslant k$ if and only if there exists a $k$-dimensional vector bundle $\mu^{k}$ such that $\mu^{k} \oplus \psi^{-}=\varepsilon^{k} \oplus \psi^{+}$. We recall that if we consider $f: M^{n} \rightarrow X^{n+k}$ to be a continuous map between two closed smooth manifolds and $\psi=f^{*} \tau_{X}-\varepsilon^{k} \oplus \tau_{M}$, then $g \operatorname{dim}(\psi) \leqslant k$ if there exists a vector bundle $\mu^{k}$ such that $\mu^{k} \oplus \varepsilon^{k} \oplus \tau_{M} \simeq \varepsilon^{k} \oplus f^{*} \tau_{X}$. This isomorphism is equivalent to $\mu^{k} \oplus \tau_{M} \simeq f^{*} \tau_{X}$, and then, by [8], $f$ is homotopic to an immersion.

In order to study whether $g \operatorname{dim}(\psi) \leqslant k$ we need to define a fibre bundle $\tilde{V}_{k}\left(\psi^{q}\right)$ over $X$. Consider the bundle Iso $\left(\varepsilon^{k} \oplus \psi^{-}, \varepsilon^{k} \oplus \psi^{+}\right) \rightarrow X$, whose fibre consists of $\operatorname{Iso}\left(\mathbb{R}^{k} \oplus\right.$ $\left.\left(\psi^{-}\right)_{x}, \mathbb{R}^{k} \oplus\left(\psi^{+}\right)_{x}\right)$. The linear group $G l_{k}$ acts freely on the right and then we define $V_{k}(\psi)=\operatorname{Iso}\left(\varepsilon^{k} \oplus \psi^{-}, \varepsilon^{k} \oplus \psi^{+}\right) / G l_{k}$, which is a fibre bundle over $X$ with fibre homotopy equivalent to a Stiefel manifold. For each $t$ we can construct $V_{k}\left(\psi^{+} \oplus \varepsilon^{t}-\psi^{-} \oplus \varepsilon^{t}\right)$ over $X$ whose fibre is also $(k-1)$-connected. Then we define

$$
\tilde{V}_{k}(\psi)=\bigcup_{t=0}^{\infty} V_{k}\left(\psi^{+} \oplus \varepsilon^{t}-\psi^{-} \oplus \varepsilon^{t}\right)
$$

over $X$ with $(k-1)$-connected fibre. Since $G l_{k}$ acts freely on $\operatorname{Iso}\left(\varepsilon^{k} \oplus \psi^{-}, \varepsilon^{k} \oplus \psi^{+}\right)$and effectively on $\mathbb{R}^{k}$, we have that $\operatorname{Iso}\left(\varepsilon^{k} \oplus \psi^{-}, \varepsilon^{k} \oplus \psi^{+}\right) \times_{G l_{k}} \mathbb{R}^{k}$ is a $k$-dimensional vector bundle $\mu^{k}$ over $\tilde{V}_{k}(\psi)[\mathbf{1 3}]$. In this paper we will consider

$$
\tilde{V}_{k}(\psi) \stackrel{\pi}{\rightarrow} X \times B O(q),
$$

with $\psi=\gamma^{q}-\varepsilon^{q}$ a virtual bundle over $X \times B O(q)$ and where $\gamma^{q}$ denotes the pull-back of the universal vector bundle over $B O(q)$, by the second projection $\pi_{2}: X \times B O(q) \rightarrow$ $B O(q)$.

Let us consider $\theta^{\prime}: \tilde{V}_{k}(\psi) \rightarrow B O(k)$, the classifying map of the vector bundle $\mu^{k}$, which is a high homotopy equivalence, for $k$ large enough.

Let $\alpha^{p}$ be an arbitrary $p$-dimensional vector bundle over $X$, and, for each $q$, consider $\phi^{p+q}=\varepsilon^{p+q}-\left(\alpha^{p} \times \gamma^{q}\right)$, a virtual bundle over $X \times B O(q)$. We note that, for $q$ large,

$$
\Omega_{n}\left(X \times B O, \phi^{p+q}\right) \simeq \pi_{n+p+q}^{S}(T(\alpha) \wedge M O),
$$

where $T(\alpha)$ is the Thom space [9] and, since $T(\alpha)$ is $(p-1)$-connected, we conclude that $\eta_{n}(X) \simeq \Omega_{n}\left(X \times B O, \phi^{p+q}\right)$ and then this normal bordism group does not depend on $\alpha^{p}$.

The following diagram is commutative:

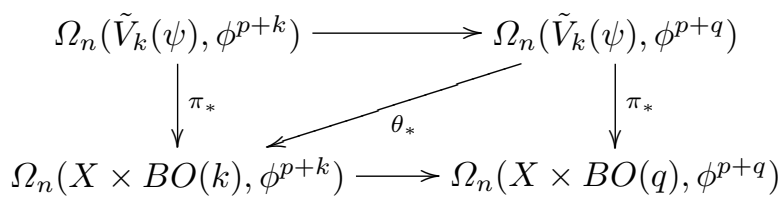

where $\theta_{*}$, induced by $\theta^{\prime}$, is an isomorphism for $q$ large, from remarks above. 
Let us suppose that $n \leqslant 2 k+2$. These identifications and Diagram (I) fit in a sequence of Salomonsen [13] yielding the following exact sequence:

$$
\begin{aligned}
(\mathrm{II}) & \longrightarrow \Omega_{n-k}\left(X \times B O(q) \times P^{\infty}, \Gamma_{k}\right) \longrightarrow I_{n}(X) \stackrel{\mathcal{F}}{\longrightarrow} \eta_{n}(X) \\
& \stackrel{\tilde{\gamma}_{k-1}}{\longrightarrow} \Omega_{n-k-1}\left(X \times B O(q) \times P^{\infty}, \Gamma_{k-1}\right) \longrightarrow \cdots,
\end{aligned}
$$

where

$$
\Gamma_{k}=\nu_{X}^{p} \times \gamma^{q} \oplus\left(\varepsilon^{q-n+k}-\gamma^{q}\right) \otimes \lambda-\varepsilon^{p+q-n+k}
$$

and $\lambda$ is the canonical bundle over the real projective space $P^{\infty}$.

Next we take $\psi$ a virtual vector bundle over $M$ and suppose that $5 \leqslant n<2 k$. Then from the exact sequence of Salomonsen [13], we have the following exact sequence:

(III) $\longrightarrow \Omega_{n}\left(\tilde{V}_{k}(\psi), \tau_{M}-\varepsilon^{n}\right) \stackrel{\pi_{M_{*}}}{\longrightarrow} \Omega_{n}\left(M, \tau_{M}-\varepsilon^{n}\right) \stackrel{\gamma_{M}}{\longrightarrow} \Omega_{n-k-1}\left(M \times P^{\infty}, \Phi\right) \longrightarrow \cdots$, where $\Phi=-(n-k-1) \lambda-\lambda \otimes \psi+\tau_{M}-\varepsilon^{n}$ and $\gamma_{M}$ is defined in the construction of the sequence (see Theorem 6.1 in $[\mathbf{1 3}]$ ).

We recall that if $\psi=h^{*} \tau_{X}-\varepsilon^{k} \oplus \tau_{M}$, where $h: M \rightarrow X$ is a continuous map, $5 \leqslant n<2 k$, then $\gamma_{M}([M])$ is the invariant $\omega_{k}\left(\nu_{h}\right)$ defined by Koschorke $[\mathbf{1 0}, \mathbf{1 1}]$, which is an obstruction to the existence of a monomorphism from $M \times \mathbb{R}^{\ell}$ into $\nu_{h}$. With this notation, $h$ is homotopic to an immersion if and only if $\gamma_{M}([M])=0$.

Here, $[M]=\left[M, 1_{M}, t_{M}\right] \in \Omega_{n}\left(M, \tau_{M}-\varepsilon^{n}\right)$ is the fundamental class of $M, t_{M}$ : $\tau_{M} \oplus \varepsilon^{n} \rightarrow \varepsilon^{n} \oplus \tau_{M}$ being the isomorphism which interchanges factors.

\section{Proofs of Theorems A and B}

Proof of Theorem A. Let $h: M \rightarrow X$ be a continuous map from a closed connected smooth $n$-dimensional manifold $M$ into a smooth connected $(n+k)$-dimensional manifold $X$.

Let us now consider the following commutative diagram, where the left-hand vertical sequence is (III) with $\psi=h^{*} \tau_{X}-\varepsilon^{k} \oplus \tau_{M}$, the right-hand vertical sequence is (II) and $(h, g)_{*}$ and $((h, g) \times \mathrm{Id})_{*}$ are induced maps of $(h, g)$ in convenient normal bordism groups:

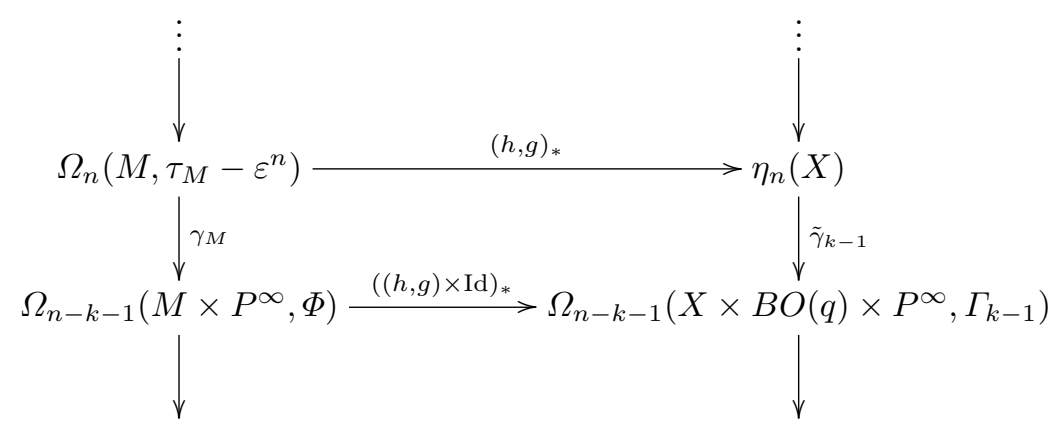

Suppose that $h$ is bordant to an immersion. Then

$$
0=\tilde{\gamma}_{k-1}([M, h])=((h, g) \times \mathrm{Id})_{*}\left(\gamma_{M}([M])\right) .
$$


Since, by assumption,

$$
(h, g)_{*}: H_{i}\left(M, \mathbb{Z}_{2}\right) \rightarrow H_{i}\left(X \times B O, \mathbb{Z}_{2}\right)
$$

is an isomorphism for $i<n-k$ and an epimorphism for $i=n-k$, we conclude that $((h, g) \times \mathrm{Id})_{*}$ is a $\mathcal{C}$-isomorphism for $i=n-k-1$ and then $\operatorname{ker}((h, g) \times \mathrm{Id})_{*} \in \mathcal{C}$.

We recall that the order of the elements of the image of $\gamma_{M}$ is a power of $2[\mathbf{9}, \mathbf{1 3}]$. Therefore, $\gamma_{M}([M, h])=0$ and $h$ is homotopic to an immersion [10].

Proof of Theorem B. We recall that under the hypotheses of Theorem B,

$$
f_{*}: \Omega_{n}\left(M, f^{*} \tau_{N}-\varepsilon^{n}\right) \rightarrow \Omega_{n}\left(N, \tau_{N}-\varepsilon^{n}\right)
$$

is a $\mathcal{C}$-isomorphism and $f^{*}\left(\beta_{2}\right)=\alpha_{2}$, where $\alpha=\nu_{M}$, and $\beta=\nu_{N}$ are the stable normal bundles of $M$ and $N$, and $\alpha_{2}$ and $\beta_{2}$ are the respective 2-localization [2].

Let us consider the following commutative diagram:

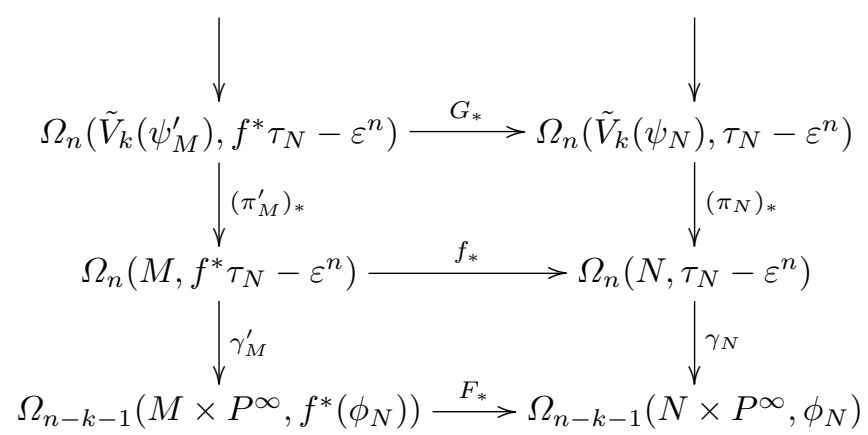

where the right-hand sequence is obtained from (III), $\psi_{N}=\varepsilon^{n+k}-\tau_{N} \oplus \varepsilon^{k}, \psi_{M}^{\prime}=$ $\varepsilon^{n+k}-f^{*} \tau_{N} \oplus \varepsilon^{k}$. The left-hand sequence is induced from the right-hand sequence by $f$ and by $G$ and $F$, which are induced by $f$ and are given in [13].

We observe that $\left(\pi_{M}^{\prime}\right)_{*}$ is the induced map of $\pi_{M}$ in normal bordism groups with virtual bundle $f^{*} \tau_{N}-\varepsilon^{n}$.

If $N$ immerses in $\mathbb{R}^{n+k}$, then $\left(\pi_{N}\right)_{*}$ is surjective [13] and, since $f_{*}: H_{i}\left(M, \mathbb{Z}_{2}\right) \rightarrow$ $H_{i}\left(N, \mathbb{Z}_{2}\right)$ is an isomorphism for $i \geqslant 0, F_{*}$ is a $\mathcal{C}$-monomorphism. Therefore, $\left(\pi_{M}^{\prime}\right)_{*}$ is a $\mathcal{C}$-epimorphism and since the order of every element of the image of $\gamma_{M}^{\prime}$ is a power of $2[\mathbf{1 3}]$, we conclude that $\left(\pi_{M}^{\prime}\right)_{*}$ is an epimorphism.

Now, we only to need to show that $\left(\pi_{M}\right)_{*}: \Omega_{n}\left(\tilde{V}_{k}\left(\psi_{M}\right), \tau_{M}-\varepsilon^{n}\right) \rightarrow \Omega_{n}\left(M, \tau_{M}-\varepsilon^{n}\right)$ is a $\mathcal{C}$-epimorphism, where $\psi_{M}=\varepsilon^{n+k}-\tau_{M} \oplus \varepsilon^{k}$. For this, we consider the commutative diagram

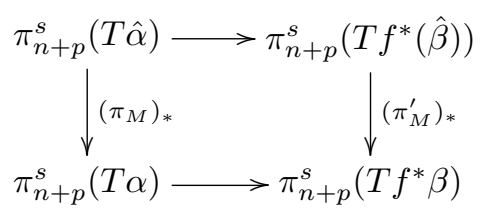

where $\hat{\beta}$ and $\hat{\alpha}$ denote the pull-back of $\beta$ and $\alpha$ by $\pi_{N}$ and $\pi_{M}$, respectively. The two horizontal maps are $\mathcal{C}$-isomorphisms $[2]$ and $\left(\pi_{M}\right)_{*}$ is a $\mathcal{C}$-epimorphism. 


\section{Applications}

Let $M$ and $N$ be closed smooth manifolds of dimension $n$ and $(n+k)$, respectively, and let $f: M \rightarrow N$ be a continuous map. Define $U_{f} \in H^{k}\left(N, \mathbb{Z}_{2}\right)$ to be the image of the fundamental class $[M] \in H_{n}\left(M, \mathbb{Z}_{2}\right)$ by the composite map

$$
H_{n}\left(M, \mathbb{Z}_{2}\right) \stackrel{f_{*}}{\longrightarrow} H_{n}\left(N, \mathbb{Z}_{2}\right) \stackrel{D_{N}^{-1}}{\longrightarrow} H^{k}\left(N, \mathbb{Z}_{2}\right),
$$

where $D_{N}$ denotes the Poincaré duality isomorphism.

We also consider the following commutative diagram:

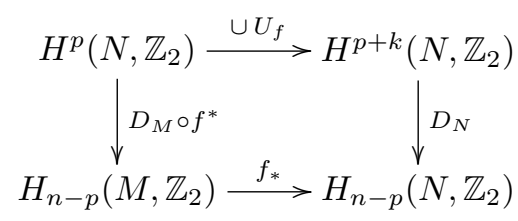

where ' $U$ ' denotes the cup product.

Theorem 4.1. Let $M$ and $N$ be closed smooth manifolds of dimension $n$. Suppose that

$$
H_{i}\left(M, \mathbb{Z}_{2}\right) \simeq H_{i}\left(N, \mathbb{Z}_{2}\right), \quad \text { for all } i \geqslant 0,
$$

and there exists $f: M \rightarrow N$ with $\operatorname{deg}_{2} f=1$. Then $f_{*}: H_{i}\left(M, \mathbb{Z}_{2}\right) \rightarrow H_{i}\left(N, \mathbb{Z}_{2}\right)$ is an isomorphism, for $i \geqslant 0$.

Proof. Since the dimension of $M$ and of $N$ is $n$, we have that $U_{f} \in H^{0}\left(N, \mathbb{Z}_{2}\right)$ and $U_{f}=\operatorname{deg}_{2} f$.

Therefore, $\cup U_{f}$ is a multiple of $\operatorname{deg}_{2} f=1$, so that

$$
\cup U_{f}: H^{p}\left(N, \mathbb{Z}_{2}\right) \rightarrow H^{p}\left(N, \mathbb{Z}_{2}\right) \text { is the identity map }
$$

for $p \geqslant 0$ and

$$
f_{*}: H_{n-p}\left(M, \mathbb{Z}_{2}\right) \rightarrow H_{n-p}\left(N, \mathbb{Z}_{2}\right) \text { is onto }
$$

for all $p \geqslant 0$. But $H_{i}\left(M, \mathbb{Z}_{2}\right) \simeq H_{i}\left(N, \mathbb{Z}_{2}\right), i \geqslant 0$, and the result follows.

Corollary 4.2. Let $M$ and $N$ be closed smooth $n$-manifolds with isomorphic homology groups. Suppose that there exists $f: M \rightarrow N$ with $\operatorname{deg}_{2} f=1$. Then $M$ immerses in $\mathbb{R}^{n+k}, 5 \leqslant n<2 k$, if and only if $N$ does.

Let $M$ and $N$ be closed smooth $n$-manifolds. Given $x_{0} \in M^{n}$ and $y_{0} \in N^{n}$, let us take $D_{1}^{n}$ and $D_{2}^{n}$ discs containing $x_{0}$ and $y_{0}$, respectively, for which there exists a homeomorphism $h: D_{1}^{n} \rightarrow D_{2}^{n}$ with $h\left(x_{0}\right)=y_{0}$.

Put $A=\partial D_{1}, M_{n-1}=M^{(n-1)} \cup A$, where $M^{(n-1)}$ is the $(n-1)$-skeleton of $M$, $Y=N-h\left(\stackrel{\circ}{D}_{1}\right), f_{0}=\left.h\right|_{A}$, and let

$$
\chi_{n}^{n-1}: H^{n}\left(M, A, \pi_{n-1}(Y)\right) \rightarrow H^{n}\left(M, A, H_{n-1}(Y)\right)
$$

be the homomorphism induced in cohomology by the Hurewicz homomorphism. 
Let us suppose that $f_{0}$ extends to $M_{n-1}, Y$ is $(n-1)$-simple and $H_{n-1}(A, \mathbb{Z})$ is a free group.

Theorem 4.3. Suppose that $M^{n}$ and $N^{n}$ are such that $H_{*}\left(M, \mathbb{Z}_{2}\right) \simeq H_{*}\left(N, \mathbb{Z}_{2}\right)$.

If $\chi_{n}^{n-1}$ is a monomorphism and there exists a homomorphism $\psi: H_{n}(M, \mathbb{Z}) \rightarrow$ $H_{n}(N, \mathbb{Z})$ such that $\left(f_{0}\right)_{*}=\psi \circ i_{*}$, with $i_{*}: H_{n}(A, \mathbb{Z}) \rightarrow H_{n}(M, \mathbb{Z})$ induced by the inclusion, then there exists $f: M \rightarrow N$ with $\operatorname{deg}_{2} f=1$.

Proof. Under these conditions, $f_{0}$ extends to $f: M \rightarrow N$ (see [1] ) with $f\left(M-\stackrel{\circ}{D}_{1}\right)=$ $N-f\left(\stackrel{\circ}{D}_{1}\right)$. By excision, $H_{n}\left(M, \mathbb{Z}_{2}\right)$ (respectively, $\left.H_{n}\left(N, \mathbb{Z}_{2}\right)\right)$ is isomorphic to $H_{n}(M, M-$ $\left.x_{0}, \mathbb{Z}_{2}\right)$ (respectively, $H_{n}\left(N, N-y_{0}, \mathbb{Z}_{2}\right)$ ), which is isomorphic to $H_{n}\left(D_{1}, D_{1}-x_{0}, \mathbb{Z}_{2}\right)$ (respectively, $H_{n}\left(f\left(D_{1}\right), f\left(D_{1}\right)-y_{0}, \mathbb{Z}_{2}\right)$ ) and the result follows.

We finish with some examples which illustrate Theorem A. In these examples, we are supposing that $h: M^{n} \rightarrow X^{n+k}$ is bordant to an immersion.

Example 4.4. Let us consider $n \geqslant 5$ and $k=n-2$. In order for

$$
(h, g)^{*}: H^{1}\left(X, \mathbb{Z}_{2}\right) \oplus H^{1}\left(B O, \mathbb{Z}_{2}\right) \rightarrow H^{1}\left(M, \mathbb{Z}_{2}\right)
$$

to be an isomorphism, one needs to take $M$ such that $w_{1}(M) \neq 0$, because otherwise $(h, g)^{*}\left(w_{1}(X)+w_{1}(\gamma)\right)=0$. For example, $M^{n}=P^{n}, n$ even, and $H^{1}\left(X, \mathbb{Z}_{2}\right)=0$.

Example 4.5. If $n \geqslant 7$ and $k=n-3$, we take $M^{n}$ as the real Grassmannian manifold $G_{l+2,2}$ with $l>3$ and $X$ sufficiently highly connected that $H^{i}\left(X \times B O, \mathbb{Z}_{2}\right)=$ $H^{i}\left(B O, \mathbb{Z}_{2}\right)$. Then, by $[\mathbf{1 2}], H^{i}\left(B O, \mathbb{Z}_{2}\right) \rightarrow H^{i}\left(G_{l+2,2}, \mathbb{Z}_{2}\right)$ is an isomorphism for $i \leqslant 3$.

Acknowledgements. The authors express their thanks to Ulrich Koschorke and Pedro Pergher for their helpful comments and many suggestions.

\section{References}

1. C. BIASI, Teoria da obstrução e aplicações, in Notas do Instituto de Ciências Matemáticas de S. Carlos-USP (1986).

2. C. Biasi, D. L. Gonçalves and A. K. M. Libardi, Immersions in the metastable dimension range via the normal bordism approach, Topology Applic. 116 (2001), 293303.

3. R. L. W. Brown, Immersions and embeddings up to cobordism, Can. J. Math. 23 (1971), 1102-1115.

4. P. E. Conner And E. E. Floyd, Differentiable periodic maps, Ergenbisse der Mathematik und ihrer Grenzgebiete, vol. 33, pp. 10-19 (Springer, 1964).

5. H. Glover AND W. Homer, Immersing manifolds and 2-equivalence, Lectures Notes in Mathematics, vol. 657, pp. 194-197 (Springer, 1978).

6. H. Glover And W. Homer, Metastable immersion, span and the two-type of a manifold, Can. Math. Bull. 29 (1986), 20-24.

7. H. Glover and G. Mislin, Immersion in the metastable range and 2-localization, Proc. Am. Math. Soc. 43 (1974), 443-448.

8. M. HiRsch, Immersions of manifolds, Trans. Am. Math. Soc. 93 (1959), 242-276.

9. U. KOSCHORKE, Vector fields and other vector bundle morphism-a singularity approach, Lecture Notes in Mathematics, vol. 847 (Springer, 1981). 
10. U. KOSCHORKE, The singularity method and immersions of m-manifolds into manifolds of dimensions $2 m-2,2 m-3$ and $2 m-4$, Lecture Notes in Mathematics, vol. 1350 (Springer, 1988).

11. U. Koschorke, Nonstable and stable monomorphisms of vector bundles, Topology Applic. 75 (1997), 261-286.

12. J. W. Milnor And J. Stashed, Lectures on characteristic classes, Ann. Math. Stud. 76 (1974), 73-81.

13. H. A. Salomonsen, Bordism and geometric dimension, Math. Scand. 32 (1973), 87-111. 